\title{
Low Energy Nucleon-Nucleon Scattering with the Skyrme Model in the Geodetic Approximation
}

\author{
by T. Gisiger and M. B. Paranjape \\ Laboratoire de physique nucléaire, Université de Montréal C.P. 6128, succ "A", Montréal, \\ Québec, Canada, НзС зJ'7
}

\begin{abstract}
We calculate nucleon-nucleon scattering at low energies and large impact parameter in the Skyrme model within the framework for soliton scattering proposed by Manton. This corresponds to a truncation of the degrees of freedom to the twelve collective coordinates which essentially describe the rigid body motion of the pair of Skyrmions. We take to its logical conclusion the result that the induced kinetic energy for these collective coordinates in the product ansatz behaves as one over the separation and hence can dominate over the potential. This behaviour implies to leading order that we can drop the potential and the resulting motion reduces simply to geodesic motion on the manifold parametrized by the variables of the product ansatz. We formulate the semi-classical quantization of these variables to obtain the motion corresponding to the nucleonic states of the Skyrme model. This is the appropriate description for the nucleons in order to consider their scattering within Manton's framework in the semi-classical approximation. We investigate the implications for the scattering of nucleons with various initial polarizations using the approximation method of "variation of constants".
\end{abstract}




\section{Introduction}

The Skyrme mode 11 is unique among models describing the strong interactions. It is conceived from the very basic and fundamental principles concerning the symmetries and their realizations in the strong interaction, and it can be used to make quantitative predictions about low energy hadronic dynamics. It contains exactly two parameters, $f_{\pi}$ and $e$, which can in principle be computed from QCD. In practice, they are fitted by phenomenological consideration. Once these parameters are fixed, the model can make reasonably accurate predictions in the meson sector 2 and for the static properties of single baryons 3 .

The case $B=2$ is very interesting since it contains both the scattering of two nucleons and their bound state, the deuteron. Much effort has been invested in extracting the nucleonnucleon potentiall. This comprises of a calculation of the potential energy of two well separated Skyrmions, taken in the product ansatz or slight variations thereof, completed with a projection onto asymptotic quantum nucleonic states. For large separation $d$ between the nucleons, the static potential behaves as $1 / d^{3}$, for the case of massless pions, and contributes only to the spin-spin and tensor channels of the nucleon-nucleon potential. Adding a pion mass cuts off all interactions with the usual Yukawa decaying exponential, however the potential now has a leading $1 / d$ behaviour in the tensor channel and a $1 / d^{2}$ central channel, albeit, both are multiplied by two powers of the pion mass. The product ansatz is deficient to produce an intermediate range attraction in the central channel, however, it has been demonstrated by exact numerical calculations unfettered by this ansatz, that the Skyrme model does indeed contain a central attraction 1 . The Skyrme model has not been too useful for determining the potential at small separation. The exact deuteron profile is thought to be

a toroidal configuration where the Skyrmions loose their identity. In this way it is clear that the product ansatz will fail to properly describe the situation, since it distinctly identifies the two Skyrmions.

It is the aim of this paper to consider the influence of the kinetic energy on the nucleonnucleon interaction. Within the framework of Manton $\mathrm{d}$ for soliton scattering, the system is 
truncated to the finite number of degrees of freedom parametrizing the low energy critical points of the static configuration and the gradient flow curves joining them together. The full potential serves to induce a potential on this sub-manifold, while the full kinetic energy serves to induce a metric. We, and also Schroer 16 have computed the induced kinetic energy for the case of massless pions 1 and found a leading term which behaves as $1 / d$. This term can dominate for large separation over the contribution from the potential which behaves as $1 / d^{3}$. We wish to investigate the effect of this kinetic term on nucleon-nucleon scattering. There are two questions to address: firstly how to properly formulate the inclusion of the term within the low energy, semi-classical framework and secondly, how to characterize its effects on the scattering. We will work with the hypothesis that the potential term is in fact negligible, we discuss in the conclusion the actual physical realization of this eventuality. With this hypothesis, the scattering corresponds to geodesic motion in the induced metric on the sub-manifold. The effect of this induced metric is to modify the equations of motion in a rather complicated way. The utility of the differential geometric formulation is less evident with respect to the corresponding work on scattering of BPS

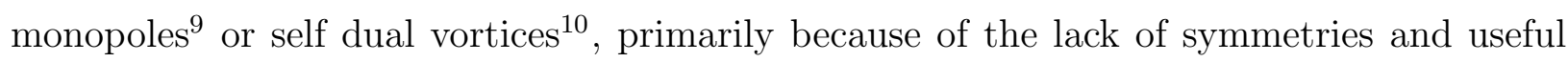
algebraic structures. Instead the equations are better formulated as one complicated, exact, dynamical equation for the coordinate corresponding to the relative separation, augmented by conservation laws, which are now violated by perturbative corrections coming from the new interaction, for the generators of rotation and for isorotation of the free system. We make a further approximation, using the method of "variation of constants" 1 to compute the scattering. The method essentially consists of using the free trajectories in the interaction terms to produce evolution of the previously conserved (constant) quantities. We find nontrivial scattering for all initial polarizations.

The extraction of the implied nucleon-nucleon scattering is non-standard. Up to now, most investigations have focussed directly on the projection onto asymptotic, quantum nucleonic states. This requires a consistent interpretation of the kinetic energy as supplying an induced metric on the sub-manifold. This metric modifies the generally covariant Lapla- 
cian defined on the sub-manifold which then should be included in the relevant Schrödinger operator 7 . We find that this procedure has not been fully implemented, 1 , 23 . It remains to be seen whether directly quantizing the truncated system is phenomenologically viable. Actually we find this procedure somewhat orthogonal to the incorporation of classical solitons within the quantum theory. This incorporation is necessarily formulated in terms of the semi-classical perturbative expansion, indeed the soliton is a classical solution. In this article we give the construction of the semi-classical nucleonic states using the Bohr-Sommerfeld (WKB) type quantization rules. These states are then amenable to calculation of the scattering within the semi-classical approximation.

In section 2 we give the equations of motion and the approximation methods used. In section 3 we give the analysis of the semi-classical quantum nucleon states. In section 4 we summarize our results for the scattering and we terminate with our conclusions in section 5 .

\section{Equations of Motion and Approximation Methods}

In this section we will indicate briefly the path to deriving the equations of motion and the approximations that we use in our treatement. For a more detailed description we refer the reader to our previous article and references therein 8 .

With the definitions

$$
\begin{aligned}
& \mathcal{L}_{\mu}^{a}(U)=-\frac{i}{2} \operatorname{tr}\left[\tau^{a} \partial_{\mu} U U^{\dagger}\right] \\
& \mathcal{R}_{\mu}^{a}(U)=-\frac{i}{2} \operatorname{tr}\left[\tau^{a} U^{\dagger} \partial_{\mu} U\right] \\
& D_{a b}(U)=\frac{1}{2} \operatorname{tr}\left[\tau^{a} U \tau^{b} U^{\dagger}\right]
\end{aligned}
$$

where the field $U(\vec{x}, t)$ is an element of the $2 \times 2$ matrix valued representation of $S U(2)$, the Skyrme Lagrangean becomes

$$
\mathcal{L}_{s k}=\frac{f_{\pi}^{2}}{2} \mathcal{L}_{\mu}(U) \cdot \mathcal{L}^{\mu}(U)-\frac{1}{4 e^{2}}\left[\mathcal{L}_{\mu}(U) \cdot \mathcal{L}^{\mu}(U) \mathcal{L}_{\nu}(U) \cdot \mathcal{L}^{\nu}(U)-\mathcal{L}_{\mu}(U) \cdot \mathcal{L}^{\nu}(U) \mathcal{L}_{\nu}(U) \cdot \mathcal{L}^{\mu}(U)\right] .
$$


We replace for $U(\vec{x}, t)$ in this Lagrangean with the product ansatz

$U(\vec{x}, t)=A U_{s}\left(\vec{x}-\vec{R}_{1}\right) A^{\dagger} B U_{s}\left(\vec{x}-\vec{R}_{2}\right) B^{\dagger}$

where $U_{s}(\vec{x})=e^{i f(r) \hat{r} \cdot \vec{\tau}}$ is the single Skyrmion with profile function $f(r), A(t), B(t) \in S U(2)$ define the respective spin and isospin orientations of the Skyrmions and $\vec{R}_{1}$ and $\vec{R}_{2}$ are their positions. This corresponds to the truncation to the relevant degrees of freedom for the low energy behaviour. We obtain the kinetic energy by keeping only the terms which contain time derivatives, these are automatically quadratic, the remaining terms contain no time derivatives and comprise the potential.

The product ansatz makes sense only for well separated Skyrmions, when they are close, we expect strong deformations and, in fact, the minimal critical point is conceived to be a toroidal configuration 14 . A proposal to globally, approximately describe the sub-manifold of low energy critical points and the union of gradient flow curves joining these together has been put forward by Atiyah and Manton 15. We do not consider, however, the possibility of close encounters between the Skyrmions and hence the product ansatz should yield at least the correct, leading asymptotic result. The induced kinetic energy has a well defined expansion in inverse powers of the separation $d$. The zero order terms correspond to the free kinetic energy of each individual Skyrmion. The leading correction behaves as $1 / d$ and contributes to a spin-spin and tensor interaction between the Skyrmions, as we $\mathrm{B}$ and Schroers 16 have found. Thus the kinetic energy is given by

$$
\begin{aligned}
T=\frac{1}{4} M \dot{\vec{d}}^{2}+ & 2 \Lambda\left(\mathcal{L}^{a}(A) \mathcal{L}^{a}(A)+\mathcal{L}^{a}(B) \mathcal{L}^{a}(B)\right) \\
& +\frac{\Delta}{d} \epsilon^{i a c} \epsilon^{j b d} \mathcal{R}^{c}(A) \mathcal{R}^{d}(B)\left(\delta^{i j}-\hat{d}^{i} \hat{d}^{j}\right) D_{a b}\left(A^{\dagger} B\right)+O\left(1 / d^{2}\right)
\end{aligned}
$$

where $M$ is the Skyrmion mass, $\Lambda$ its moment of inertia, $\Delta=2 \pi \kappa^{2} f_{\pi}^{2}, F(r) \sim \kappa / r^{2}$ at large $r, \mathcal{L}^{a}(A) \equiv \mathcal{L}_{0}^{a}(A), \mathcal{R}^{a}(A) \equiv \mathcal{R}_{0}^{a}(A)$ and $\hat{d}=\vec{d} / d=\left(\vec{R}_{1}-\vec{R}_{2}\right) / d$. The potential has been calculated by many authors] 17 22. It has a leading term which contributes to the tensor channel and behaves as $1 / d^{3}$, apart from the "free" contribution which gives the mass of each Skyrmion: 
$V=2 M+4 \pi f_{\pi}^{2} \kappa^{2} \frac{(1-\cos \theta)\left(3(\hat{n} \cdot \hat{d})^{2}-1\right)}{d^{3}}$.

The complete Lagrangean of the system is given by

$L=T-V$.

In this paper we focus on the effects of the kinetic term. The equations of motion arising from the kinetic term are neither illuminating nor practical. It is useful just to write down the approximate equations. The free Lagrangean is completely integrable and corresponds exactly to two free, spherically symmetric tops. We can express the approximate equations as corrections to conservation laws using the approximation of Lagrange 11 . Here we take the Poisson brakets implied by the free dynamics to compute the corrections implied by the interaction to the equations of motion. This yields a practical set of equations which must be treated, however, with further perturbation measures

$$
\begin{aligned}
& \frac{d}{d t} \dot{d}^{k}=-\frac{2 \Delta}{M d^{2}}\left[\delta^{i j} \hat{d}^{k}+\delta^{j k} \hat{d}^{i}+\delta^{i k} \hat{d}^{j}-3 \hat{d}^{i} \hat{d}^{j} \hat{d}^{k}\right] \epsilon^{i a c} \epsilon^{j b d} \mathcal{R}^{c}(A) \mathcal{R}^{d}(B) D_{a b}\left(A^{\dagger} B\right) \\
& \frac{d}{d t} \mathcal{L}^{k}(A)=\frac{\Delta}{2 M d} \epsilon^{i a c} \epsilon^{j b d} \mathcal{R}^{c}(A) \mathcal{R}^{d}(B)\left(\delta^{i j}-\hat{d}^{i} \hat{d}^{j}\right) \epsilon^{k e f} D_{f a}(A) D_{e b}(B) \\
& \frac{d}{d t} \mathcal{L}^{k}(B)=\frac{\Delta}{2 M d} \epsilon^{i a c} \epsilon^{j b d} \mathcal{R}^{c}(A) \mathcal{R}^{d}(B)\left(\delta^{i j}-\hat{d}^{i} \hat{d}^{j}\right) \epsilon^{k e f} D_{a e}\left(A^{\dagger}\right) D_{f b}(B) \\
& \frac{d}{d t} \mathcal{R}^{k}(A)=-\frac{\Delta}{2 M d} \epsilon^{i a c} \epsilon^{j b d} \mathcal{R}^{d}(B)\left(\delta^{i j}-\hat{d}^{i} \hat{d}^{j}\right)\left[\epsilon^{k c f} \mathcal{R}^{f}(A) D_{a b}\left(A^{\dagger} B\right)+\epsilon^{k a f} D_{f b}\left(A^{\dagger} B\right) \mathcal{R}^{c}(A)\right] \\
& \frac{d}{d t} \mathcal{R}^{k}(B)=-\frac{\Delta}{2 M d} \epsilon^{i a c} \epsilon^{j b d} \mathcal{R}^{c}(A)\left(\delta^{i j}-\hat{d}^{i} \hat{d}^{j}\right)\left[\epsilon^{k d f} \mathcal{R}^{f}(B) D_{a b}\left(A^{\dagger} B\right)+\epsilon^{k b f} D_{a f}\left(A^{\dagger} B\right) \mathcal{R}^{d}(B)\right] .
\end{aligned}
$$

The method of variation of constants is ideal for the following analysis. Here we replace the variables in the right hand side of the "conservation equations" by their trajectories coming from the free dynamics. This gives rise to a "variation" of the previously conserved "constants". The procedure can be iterated to give higher order corrections. One should however always maintain consistency with the first (Lagrange) approximation. In fact, the method of "variation of constants" is only useful with respect to the equation for $\vec{d}$, where it gives the scattering trajectory. The change in the spin or isospin governed by the subsequent four equations cannot be used in conjunction with this approximation method because of the 
long range of the interaction. The results give an infinite change in these angular momenta, which is not reliable.

\section{Semiclassical Nucleon states}

In this section we will construct quantum states corresponding to the nucleons, using the semi-classical Bohr-Sommerfeld quantization rules applied to the classical motion of the free Skyrmion. To apply these rules, we must find the classical action-angle variables of the system and impose quantization conditions $18,1 \mathrm{1}$. This enables us to treat a Skyrmion as a nucleon consistently within the context of the semi-classical expansion. Going directly to the quantization of the Skyrmion 3 , 22 is perhaps not in the spirit of the semi-classical formulation which is, we believe, incumbent upon us once we have begun to consider classical solutions. In addition, we find that with the proper semi-classical quantization we can make

physical sense of the specific, classical Skyrmion scattering trajectories for the scattering of nucleons. We start with the Lagrangean for a single Skyrmion in the center of mass frame which is given by:

$$
\begin{aligned}
L= & -M+\lambda \operatorname{tr}\left[\dot{A}^{\dagger} \dot{A}\right] \\
& =-M+2 \lambda \sum_{i=0}^{3} \dot{a}_{i}^{2}
\end{aligned}
$$

where $\dot{a}_{i}$ is the time derivative of the $a_{i}$ from the parametrization $A=a_{0}+i \vec{a} \cdot \vec{\tau}$ with $a_{0}^{2}+\vec{a}^{2}=1$ of the matrix defining the rotational characteristics of the Skyrmion. Following the treatment of the motion of a rigid body in space, we go to the system of coordinates corresponding to the usual Euler angles:

$A=e^{-i \alpha \tau_{3} / 2} e^{-i \beta \tau_{2} / 2} e^{-i \gamma \tau_{3} / 2}$

where $\alpha, \gamma \in[0,2 \pi]$ and $\beta \in[0, \pi]$. The $a_{i}$ are related to the Euler angles by the equations: 


$$
\begin{aligned}
& a_{0}=\cos \left(\frac{\beta}{2}\right) \cos \left(\frac{\alpha+\gamma}{2}\right) \\
& a_{1}=\sin \left(\frac{\beta}{2}\right) \sin \left(\frac{\alpha-\gamma}{2}\right) \\
& a_{2}=-\sin \left(\frac{\beta}{2}\right) \cos \left(\frac{\alpha-\gamma}{2}\right) \\
& a_{3}=-\cos \left(\frac{\beta}{2}\right) \sin \left(\frac{\alpha+\gamma}{2}\right) .
\end{aligned}
$$

Replacing them into the Lagrangean (10) we get

$L=-M+\frac{1}{2} \lambda\left[\dot{\alpha}^{2}+\dot{\beta}^{2}+\dot{\gamma}^{2}+2 \dot{\alpha} \dot{\gamma} \cos \beta\right]$

which is the well known Lagrangean for rigid body motion. In this case, the Euler angles are used to describe the motion of the body fixed axes relative to the laboratory fixed axes. In our case, the identification of the Euler angles will be completely different.

The action-angle variables $J_{i}$ are defined by 2

$J_{i}=\oint p_{i} d q_{i}$

where $p_{i}$ is the momentum conjugate to the coordinate $q_{i}$, and the integral is taken along a closed path followed by the system during one period in the plane $\left(q_{i}, p_{i}\right)$ of phase space. In our case there are three such variables, one for each angle. Due to the cyclic nature of the angles $\alpha$ and $\gamma, J_{\alpha}$ and $J_{\gamma}$ are readily computed:

$J_{\alpha}=\oint p_{\alpha} d q_{\alpha}=2 \pi p_{\alpha}=2 \pi \lambda[\dot{\alpha}+\dot{\gamma} \cos \beta]$

$J_{\gamma}=\oint p_{\gamma} d q_{\gamma}=2 \pi p_{\gamma}=2 \pi \lambda[\dot{\gamma}+\dot{\alpha} \cos \beta]$

In contrast, $J_{\beta}$ is much more complicated for general orbits, however, for the type of motions that we will consider it is zero. The motion of a free Skyrmion corresponds simply to uniform isorotation about a fixed axis. The equations of motion of the Lagrangean (13) are

$$
\begin{aligned}
& \ddot{\alpha}+\ddot{\gamma} \cos \beta-\dot{\gamma} \dot{\beta} \sin \beta=0 \\
& \ddot{\beta}+2 \dot{\alpha} \gamma=0 \\
& \ddot{\gamma}+\ddot{\alpha} \cos \beta-\dot{\alpha} \dot{\beta} \sin \beta=0
\end{aligned}
$$


Without loss of generality we choose the axis of isorotation to be the third axis in isospace. Then the time evolution is clearly given by linear time dependence of $\alpha$ and/or $\gamma$ with $\beta=0$ or $\pi$.

The Bohr-Sommerfeld quantization condition, derived by path integral methods 18.19 is

$W=\sum_{i} J_{i}=(n+\xi) h, \quad n=0, \pm 1, \pm 2, \cdots$

$\xi$ is a correction factor arising from the functional integral over Gaussian fluctuations about the classical trajectory. In zero order 18 we can neglect $\xi$. Then we find

$J_{\alpha}+J_{\gamma}=n h, \quad n=0, \pm 1, \pm 2, \cdots$

in perfect accord with the original Bohr-Sommerfeld quantization rules. In order to precisely fix the time dependence of $\alpha$ and $\gamma$ corresponding to nucleon states we must make reference to the isospin and spin generators. These are given by, respectively:

$$
\begin{gathered}
\hat{\mathcal{L}}^{3}=2 \lambda \mathcal{L}^{3}=-\frac{J_{\alpha}}{2 \pi} \\
\hat{\mathcal{R}}^{3}=2 \lambda \mathcal{R}^{3}=-\frac{J_{\gamma}}{2 \pi}
\end{gathered}
$$

and satisfy the usual angular momentum algebra. The symmetries of the Skyrmion dictate that spin is equal to isospin, thus

$$
\left|J_{\alpha}\right|=\left|J_{\gamma}\right|
$$

Then applying the quantization condition with $n=1$, gives

$\left|\hat{\mathcal{L}}^{3}\right|=\left|\hat{\mathcal{R}}^{3}\right|=\frac{1}{2}$

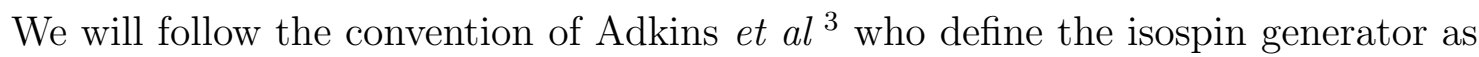

$I_{3}=-\hat{\mathcal{L}}^{3}$

and the spin generator as

$J_{3}=\hat{\mathcal{R}}^{3}$ 
Consider first the case $\beta=0$. Replacing this in equation (15), (16), (20), (23) and (24) we get

$$
\begin{aligned}
& I_{3}=\lambda(\dot{\alpha}+\dot{\gamma}) \equiv 2 \lambda \omega= \pm 1 / 2 \\
& J_{3}=-\lambda(\dot{\alpha}+\dot{\gamma}) \equiv-2 \lambda \omega=\mp 1 / 2
\end{aligned}
$$

with $(\alpha+\gamma) / 2=\phi(t)=\omega t+\phi_{0}$. This type of angular motion produces Skyrmions with spin and isospin antiparallel, and eventually the states $\mid p \downarrow>$ and $\mid n \uparrow>$. The proton state corresponds to isospin $+1 / 2$ along the 3 axis in isospace while the neutron corresponds to isospin $-1 / 2$. The selection of the third axis, we emphasize, is purely convention. States polarized along some other axis in isospace simply corresponds to linear superpositions of our states. The corresponding beams would be a mixture of protons and neutrons.

Replacing this solution in equation (12) gives

$$
\begin{aligned}
A & =\cos \phi(t)-i \sin \phi(t) \tau^{3} \\
& =e^{-i \phi(t) \tau^{3} / 2}
\end{aligned}
$$

where $\omega>0$ corresponds to the state $\mid p \downarrow>$ and $\omega<0$ to $\mid n \uparrow>$.

For $\beta=\pi$

$$
\begin{aligned}
& I_{3}=\lambda(\dot{\alpha}-\dot{\gamma}) \equiv 2 \lambda \dot{\omega}= \pm 1 / 2 \\
& J_{3}=-\lambda(\dot{\gamma}-\dot{\alpha}) \equiv 2 \lambda \dot{\omega}= \pm 1 / 2
\end{aligned}
$$

with $(\alpha-\gamma) / 2=\psi(t)=\omega t+\psi_{0}$ and the corresponding matrix

$$
\begin{aligned}
A & =i\left[\sin \psi(t) \tau^{1}-\cos \psi(t) \tau^{2}\right] \\
& =-i e^{-i \psi(t) \tau^{3} / 2} \tau^{2} e^{i \psi(t) \tau^{3} / 2}
\end{aligned}
$$

represents the state $\mid p \uparrow>$ with $\omega>0$ and $\mid n \downarrow>$ with $\omega<0$. We can see the similarity of the forms for the matrices $A$ corresponding to nucleon states and the wave functions obtained in the directly quantum version of Adkins et al 3 . In the next section we study the scattering of the nucleons as described by these states, using the geodesics approximation. 


\section{Nucleon-Nucleon scattering}

The nucleon states found in the previous section are ideally suited for our treatment of nucleon-nucleon scattering using the approximation method of the "variation of constants". We simply replace into the right hand side of equation (9) the quantized classical trajectories found in the previous section. To calculate the change induced in the previously constant quantities we integrate the equations from $t=-\infty$ to $t=+\infty$ over one free trajectory. This is useful for the equation governing the relative motion (9). For the angular variables the perturbation method is too crude. It is perhaps a reasonable perturbative scheme locally in time, however, to integrate the motion over all time leads to infinite changes in the various angular momenta. We do not trust such results and we suggest that the approximation should be iterated several times to obtain a better approximation to the local (in time) perturbation of the free trajectories. This could then perhaps be integrated over all time. At the root of the divergence is the long range $(1 / d)$ nature of the interaction term. Clearly for a physical theory with non-zero pion mass, this interaction would be cut-off by the usual Yukawa factor, rendering all such integrations finite. Thus we will consider the variation of the angular momenta only in so far as making the observation that the equations of motion with the interaction imply non-trivial spin flip and charge flip scattering. These effects are compatible with the exchange of charge carrying (pions) and spin carrying (vector mesons) intermediate particles. The first equation of (9) which describes the relative position is of course sufficient to obtain the exclusive scattering. We present below the results for the scattering of specially polarized nucleons, described in our semi-classical formalism.

It is interesting to observe that in our formalism an additional parameter arises which describes the initial state of two incoming, polarized nucleons. This parameter, along with the impact parameter, the initial velocity and the direction of polarization, actually selects the particular scattering trajectory followed by the nucleons. The parameter describes the relative orientation of the Skyrmions at a fixed time. It plays in fact a role similar to a hidden variable. An incoming pair of physical nucleons, in our formalism, has a fixed value 
for this parameter, which is only "measured" after the scattering takes place. In a physical experiment consisting of incoming beams of nucleons giving rise to collisions or scattering of pairs of nucleons, the value of this parameter will be uniformly distributed. A similar parameter arises in the case of the scattering of BPS monopolest.

There is an immediate separation of the scattering into two cases, depending on whether $D_{a b}\left(A^{\dagger} B\right)$ is time independent or not. When it depends on time for large values of the ratio $(\omega \gamma / v)$ there is an exponential suppression of the scattering where $v$ is the relative velocity and $\gamma$ the impact parameter. This is quite evident, for slowly translating Skyrmions, the prescribed rotations imposed by selecting semi-classically quantized nucleon states have the effect of averaging the interaction to zero.

We find the following expression for time variations of the previously constant relative momentum $\vec{p}=(M / 2) \dot{\vec{d}}$ :

\section{$D_{a b}\left(A^{\dagger} B\right)$ time independent:}

$$
\begin{aligned}
& p \uparrow p \uparrow \\
& \begin{array}{l}
n \downarrow n \downarrow \\
p \downarrow p \downarrow
\end{array} \quad \frac{d}{d t} p^{k}=-\frac{\Delta \omega^{2}}{d^{2}} \cos (2 \delta) \hat{d}^{k} \\
& n \uparrow n \uparrow \\
& p \uparrow p \downarrow \quad \frac{d}{d t} p^{k}=-\frac{\Delta \omega^{2}}{d^{2}}\left[\hat{d}^{k}+4 r^{k} \hat{r} \cdot \hat{d}-6 \hat{d}^{k}(\hat{r} \cdot \hat{d})^{2}\right] \\
& \hat{r}^{k}=(-\sin (\delta), \cos (\delta), 0) \\
& n \uparrow n \downarrow \quad \frac{d}{d t} p^{k}=-\frac{\Delta \omega^{2}}{d^{2}}\left[\hat{d}^{k}+4 r^{k} \hat{r} \cdot \hat{d}-6 \hat{d}^{k}(\hat{r} \cdot \hat{d})^{2}\right] \\
& \hat{r}^{k}=(\sin (\delta),-\cos (\delta), 0)
\end{aligned}
$$

\section{$D_{a b}\left(A^{\dagger} B\right)$ time dependent:}

$$
\begin{aligned}
& p \uparrow n \downarrow \\
& p \downarrow n \uparrow
\end{aligned} \quad \frac{d}{d t} p^{k}=\frac{\Delta \omega^{2}}{d^{2}} \cos (4 \omega t+\epsilon) \hat{d}^{k}
$$




$$
\begin{aligned}
& p \uparrow n \uparrow \quad \frac{d}{d t} p^{k}=\frac{\Delta \omega^{2}}{d^{2}}\left[\hat{d}^{k}+4 r^{k} \hat{r} \cdot \hat{d}-6 \hat{d}^{k}(\hat{r} \cdot \hat{d})^{2}\right] \\
& \hat{r}^{k}=(-\sin (2 \omega t+\epsilon), \cos (2 \omega t+\epsilon), 0) \\
& p \downarrow n \downarrow \quad \frac{d}{d t} p^{k}=\frac{\Delta \omega^{2}}{d^{2}}\left[\hat{d}^{k}+4 r^{k} \hat{r} \cdot \hat{d}-6 \hat{d}^{k}(\hat{r} \cdot \hat{d})^{2}\right] \\
& \hat{r}^{k}=(-\sin (2 \omega t+\epsilon),-\cos (2 \omega t+\epsilon), 0)
\end{aligned}
$$

with $\vec{d}(t)$ in the right hand side given by

$\left(d_{x}, d_{y}, d_{z}\right)=(v t, \gamma, 0)$

$A(t)$ and $B(t)$ are given by the angular dependence found in the previous section corresponding to the associated nucleon states, with $\delta=\phi_{0}^{A}-\phi_{0}^{B}$ and $\epsilon=\phi_{0}^{A}+\phi_{0}^{B}$ in self evident notation. The right hand sides can be interpreted, at this level of our approximation, as coming from a spin-spin channel and a tensor channel interaction. We stress that this is only a correspondance, the true effect of the kinetic term is to supply a non-trivial connection in the geodesic equations on the low energy sub-manifold and not to modify the potential.

To find the actual change in $\vec{p}$ and hence the scattering angle, we integrate these equations from $t=-\infty$ to $t=+\infty$. The expressions for the scattering of $p$ on $n$ each contain a time dependent $A^{\dagger} B$. When integrated these yield an exponentially suppressed variation in the dimensionless group $(\omega \gamma / v)$

$$
\sim e^{-\left(\frac{\omega \gamma}{v}\right)}
$$

Thus in the limit $v \rightarrow 0$ we get negligible scattering in these cases. For the cases $p p$ or $n n$ we find the scattering angle depends on a variable $\delta$ which corresponds to the phase lag between the rotation of $A(t)$ and $B(t)$.

We give the $p \uparrow p \uparrow$ scattering in detail to make things concrete. Here we find

$$
\begin{aligned}
p^{k}(t)=- & \Delta \omega^{2} \cos (2 \delta) \int_{-\infty}^{t} \frac{v^{k} t+\gamma^{k}}{\left(v^{2} t^{2}+\gamma^{2}\right)^{3 / 2}} d t+\frac{M}{2} v^{k} \\
= & -\Delta \omega^{2} \cos (2 \delta)\left(-\frac{v^{k}}{v^{2}\left(v^{2} t^{2}+\gamma^{2}\right)^{1 / 2}}+\frac{\gamma^{k} t}{\gamma^{2}\left(v^{2} t^{2}+\gamma^{2}\right)^{1 / 2}}\right)+\frac{M}{2} v^{k} .
\end{aligned}
$$

This yields 
$p^{k}(+\infty)=-2 \Delta \omega^{2} \cos 2 \delta \frac{\gamma^{k}}{\gamma^{2} v}+\frac{M}{2} v^{k}$

from which we calculate the cosine of the scattering angle

$$
\begin{aligned}
\cos \theta= & \frac{\vec{p}(+\infty) \cdot \vec{p}(-\infty)}{|\vec{p}(+\infty)||\vec{p}(-\infty)|} \\
& =\frac{M \gamma v^{2}}{4 \Delta} \frac{1}{\left(\frac{M^{2} \gamma^{2} v^{4}}{16 \Delta^{2}}+\omega^{4} \cos ^{2} 2 \delta\right)^{1 / 2}}
\end{aligned}
$$

This is the first analytical calculation of nucleon-nucleon scattering from essentially first

principles, without recourse to ad hoc models or potentials. We reemphasize that the Skyrme model is in principle derivable from QCD and $f_{\pi}$ and $e$ are, as such, calculable parameters and, in that sense this is also a QCD calculation.

We wish to point out that in the limit that the initial velocity vanishes, for fixed $\omega$ and $\gamma$, we recover $90^{\circ}$ scattering. This is, however, not so surprising as it is a property also shared by the Coulomb and many other interactions treated with our approximation. $90^{\circ}$ scattering is hardly remarkable except at zero impact parameter, where of course, it is impossible to avoid the region of close proximity of the nucleons and it seems important that the configurations pass through the minimal, toroidal configuration.

We have up to now considered scattering in the $(x, y)$ plane with spin polarized in the orthogonal $z$ direction. The tensorial nature of the interacton implies that the forces depend on the angle between the axis of separation and spin polarization. If we choose the spin polarization along an axis tilted with respect to the normal to the initial scattering plane we get complicated, three dimensional scattering trajectories.

\section{Conclusions}

In this paper we have taken to its logical conclusion the study of nucleon-nucleon scattering in the Skyrme model using the method of truncation to the relevant finite number of degrees of freedom and their ensuing dynamics as implied by an induced metric and potential. We have restricted ourselves to large separations between the Skyrmions. In this regime, the Skyrmions are well described to leading order by the product ansatz and the manifold of 
collective coordinates is parametrized by the variables of the product ansatz. The induced metric, calculated to leading order behaves as $1 / d$ while the induced potential behaves as $1 / d^{3}$. In principle there can be a region where the induced metric contribution dominates and we can neglect the potential. It is exactly this point of view which we have taken to its logical conclusion. We find that the metric induces an interaction which can be interpreted, within our approximation method, as a spin-spin and a tensor interaction. Unfortunately it seems that the domination by the metric term is not physically realized. The induced kinetic term is multiplied by essentially the frequencies of angular rotation of the Skyrmions while the potential term has two extra powers of the separation in the denominator. Thus for the kinetic term to dominate, the frequencies should be much larger than the separation, in units where distance, time and energies are all measured in $\mathrm{MeV}$. The frequencies are fixed by the Bohr-Sommerfeld quantization rules which give

$\omega \sim 100 \mathrm{MeV}$

This corresponds to a region of validity for a separation of about $3 \mathrm{fm}$ and greater. However there is much latitude available since the values of $f_{\pi}$ and $e$ which go into determining $\omega$ are fixed only by choosing two experimental inputs. $f_{\pi}$, e can vary as much as $10-30 \%$ thus we do not feel overly concerned with exceeding the regime of validity. Our approximation would of course be better justified for the case of $\Delta-\Delta$ scattering where $\omega \sim 300 \mathrm{MeV}$ corresponding to a separation of $1 \mathrm{fm}$. In any case, we do believe however, that it is not physically reasonable to consider the scattering of nucleons with the metric term alone and we expect a contribution from the potential term which is of the same order of magnitude. We do not expect, however, any great, qualitative modification of the scattering upon inclusion of the potential term, it is of similar strength but actually contributes only in the tensor channel for the case of massless pions.

We find our work as a prescriptive and requisite endeavour to investigate the nucleonnucleon interaction arising from the kinetic term. We have shown how to properly formulate the nucleon states within the semi-classical approximation. We have treated the scattering 
and computed the scattering angles in a systematic perturbative method. Future work should include consideration of a non-zero pion mass, which leads to a central channel interaction, a better control of the perturbation method, a departure from the product ansatz and a proper treatment of the region of close proximity, to test the validity of our formalism in the phenomenology of low energy nucleon-nucleon scattering and of the static quantum states in this sector.

We thank D. Caenepeel, J. LeTourneux, R. Mackenzie, J. M. Pearson and M. Temple-Raston for useful discussions. This work supported in part by NSERC of Canada and FCAR of Québec. 


\section{References}

1. T.H.R. Skyrme, Proc. Roy. Soc. Lon. $\underline{260}, 127$ (1961)

2. B. W. Lee, Chiral Dynamics (Gordon \& Breach, London, 1972)

3. G. S. Adkins, C. R. Nappi, E. Witten, Nucl. Phys. B228, 552, (1983)

4. A. Jackson, A. D. Jackson, V. Pasquier Nucl. Phys. A432, 567 (1985)

5. T.S. Walhout, J. Wambach, Int. Jour. Mod. Phys. E1, 665 (1992)

6. T.S. Walhout, J. Wambach, Phys. Rev. Lett. 67, 314 (1991)

7. N.S. Manton, Phys. Rev. Lett. 60, 1916 (1988)

8. T. Gisiger, M. B. Paranjape Preprint UDEM-LPN-TH-94-187, hep-th/9401040 T. Gisiger, M.B. Paranjape, Skyrmion-Skyrmion Scattering, 22nd Conference on Differential Geometrical Methods in Theoretical Physics, Ixtapa, Mexico, September, 1993, UDEM-LPN-TH-93-176, Sep 1993, hep-th/9310050.

9. G.W. Gibbons, N. S. Manton, Nucl. Phys. B274, 183 (1986)

10. T.M. Samols, Comm. Math. Phys. $\underline{145}, 149$ (1992)

11. Classical Mechanics, H. Goldstein, Second edition, Addison-Wesley publishing company 1980,505

12. E. M. Nyman, D. O. Riska, Phys. Lett. B203, 13 (1988)

13. Z. Hlousek, Int. J. Mod. Phys. A3 2681 (1988)

14. E. Braaten, L. Carson, Phys. Rev. D38, 3525 (1988); Phys. Rev. D39, 838 (1989)

V. B. Kopeliovich, B. E. Shtern, JETP Lett. 45, 203 (1987)

N. S. Manton, Phys.Rev. B192, 177 (1987)

J.J.M. Verbaarschot, T.S. Walhout, J. Wambach, H.W. Wyld, Nucl.Phys. A461 603 
$(1986)$

S. V. Zenkin, V. B. Kopeliovich, B. E. Shtern, Sov. J. Nucl. Phys. 45, 106 (1987)

15. M.F. Atiyah, N.S. Manton, Commun. Math. Phys. $\underline{153}, 3911993$.

16. B.J. Schroers (Durham U.), DTP-93-29, hep-ph/9308236

17. K. Isler, J. LeTourneux, M. B. Paranjape, Phys. Rev. D43, 43 (1991)

K. Isler, J. LeTourneux, M. B. Paranjape, Phys. Rev. D40, 2490 (1989)

18. R. Dashen, B. Hasslacher, A. Neveu, Phys. Rev. D10, 4114 (1974)

Gauge Theories and Modern Field Theory, R. Dashen, MIT Press, 1976, 403

19. Solitons and Instantons: An Introduction to Solitons and Instantons in Quantum Field Theory, R. Rajaraman, First edition, North-Holland Personal Library 1982, 186

20. Classical Mechanics, H. Goldstein, Second edition, Addison-Wesley publishing company 1980,463

21. M. Temple-Raston, D. Alexander, Nucl.Phys. B397, 195 (1993)

M. Temple-Raston, Nucl.Phys. B313, 447 (1989)

M. Temple-Raston, Phys. Lett. B206, 503 (1988)

22. R. Vinh Mau, M. Lacombe, B. Loiseau, W. N. Cottingham, P. Lisboa, Phys. Lett. B150, 259 (1985) 